\title{
Highly viscous guar gum shifts dietary amino acids from metabolic use to fermentation substrate in domestic cats
}

\author{
Kristel Rochus ${ }^{1 *}$, Geert P. J. Janssens ${ }^{1}$, Hannelore Van de Velde ${ }^{1}$, Adronie Verbrugghe ${ }^{1}$, Birgitte Wuyts ${ }^{2}$, \\ Lynn Vanhaecke $\mathrm{V}^{3}$ and Myriam Hesta ${ }^{1}$ \\ ${ }^{1}$ Laboratory of Animal Nutrition, Department of Nutrition, Genetics and Ethology, Faculty of Veterinary Medicine, \\ Ghent University, Heidestraat 19, B-9820 Merelbeke, Belgium \\ ${ }^{2}$ Laboratory of Metabolic Disorders, University Hospital Ghent, De Pintelaan 185, B-9000 Gent, Belgium \\ ${ }^{3}$ Laboratory of Chemical Analysis, Department of Veterinary Public Health and Food Safety, \\ Faculty of Veterinary Medicine, Ghent University, Salisburylaan 133, B-9820 Merelbeke, Belgium
}

(Submitted 23 January 2012 - Final revision received 25 April 2012 - Accepted 7 June 2012 - First published online 9 August 2012)

\begin{abstract}
The present study evaluated the potential of affecting amino acid metabolism through intestinal fermentation in domestic cats, using dietary guar gum as a model. Apparent protein digestibility, plasma fermentation metabolites, faecal fermentation end products and fermentation kinetics (exhaled breath hydrogen concentrations) were evaluated. Ten cats were randomly assigned to either guar gum- or cellulosesupplemented diets, that were fed in two periods of 5 weeks in a crossover design. No treatment effect was seen on fermentation kinetics. The apparent protein digestibility $(P=0.07)$ tended to be lower in guar gum-supplemented cats. As a consequence of impaired smallintestinal protein digestion and amino acid absorption, fermentation of these molecules in the large intestine was stimulated. Amino acid fermentation has been shown to produce high concentrations of acetic and butyric acids. Therefore, no treatment effect on faecal propionic acid or plasma propionylcarnitine was observed in the present study. The ratio of faecal butyric acid:total SCFA tended to be higher in guar gum-supplemented cats $(P=0.05)$. The majority of large-intestinal butyric acid is absorbed by colonocytes and metabolised to 3-hydroxy-butyrylcoenzyme A, which is then absorbed into the bloodstream. This metabolite was analysed in plasma as 3-hydroxy-butyrylcarnitine, which was higher $(P=0.02)$ in guar gum-supplemented cats. In all probability, the high viscosity of the guar gum supplement was responsible for the impaired protein digestion and amino acid absorption. Further research is warranted to investigate whether partially hydrolysed guar gum is useful to potentiate the desirable in vivo effects of this fibre supplement.
\end{abstract}

Key words: Acylcarnitine: Guar gum: Exhaled breath hydrogen: Apparent protein digestibility

Guar gum is a polysaccharide extracted from the endosperm of guar plant seeds. The guar plant (Cyamopsis tetragonolobus) is an annual leguminous plant bearing pods with round light brown seeds ${ }^{(1-3)}$. Guar gum consists of galactomannans, polysaccharides that are inert for digestive enzymes in the human small intestine ${ }^{(3)}$. In cats, the in vivo effects of guar gum intake have been investigated in blends with other fibre sources ${ }^{(4)}$, but never as the unique source of soluble fermentable fibre. In vitro research demonstrated that guar gum generated high concentrations of propionic acid upon fermentation with feline faecal inoculum ${ }^{(4,5)}$. However, further in vivo research on the end product profile upon guar gum fermentation is crucial, as recent work by Verbrugghe et al. ${ }^{(6-8)}$ suggested an amino acid-sparing effect of propionic acid in domestic cats fed fructan-supplemented diets. In strict carnivores, amino acids are continuously processed to yield glucose via the gluconeogenic pathway ${ }^{(9-11)}$. Propionic acid may be used as an alternative gluconeogenic substrate ${ }^{(12)}$, and as a consequence, amino acids might be spared. The major goal of the treatment of patients with hepatic ${ }^{(13)}$ and renal failure ${ }^{(14)}$, for example, is decreasing amino acid decarboxylation. To achieve this decrease, the amino acid-sparing potential of propionic acid might be advantageous. Also, in healthy cats, a more efficient amino acid metabolism might be beneficial, as the maintenance protein requirement for cats is higher compared to herbivorous or omnivorous species ${ }^{(10,15)}$.

The present study used dietary guar gum to evaluate whether intestinal volatile fatty acid production - in particular propionic acid - affects amino acid metabolism in domestic cats. Therefore, an in vivo assessment of guar gum fermentation metabolites in plasma, end product profiles in faeces, and kinetics was performed, and the effect of guar

Abbreviations: AUC, area under the curve; HMG, 3-OH-3-methylglutarylcarnitine; ppm, part per million.

*Corresponding author: K. Rochus, fax +329264 78 48, email kristel.rochus@ugent.be 
Table 1. Macronutrient composition of the experimental diet ${ }^{\star}$ and the experimental diet supplemented with guar gum† or celluloseł

\begin{tabular}{lrcc}
\hline Nutrient & Diet & Diet + guar gum† & Diet + cellulosef \\
\hline DM (\% as is) & 94.7 & 93.5 & 93.7 \\
Crude protein (\% DM) & 30.1 & 29.0 & 28.2 \\
Crude fat (\% DM) & 11.5 & 11.3 & 11.6 \\
Crude ash (\% DM) & 10.8 & 10.5 & 10.5 \\
Crude fibre (\% DM) & 1.4 & 2.0 & 4.0 \\
NFE (\% DM)§ & 46.2 & 47.2 & 45.7 \\
TDF (\% DM) & 8.0 & 10.9 & 10.2 \\
\hline
\end{tabular}

NFE, nitrogen-free extract; TDF, total dietary fibre.

* Labelled ingredient composition: rice, lamb meal, oils and fats, minerals and natural antioxidants.

† Vidogum GI, Unipektin Ingredients AG.

$\ddagger$ Arbocel BWW 40, Rettenmaier und Söhne.

$\S$ NFE (\% DM) was calculated as 100 - crude protein - crude fat - crude ash crude fibre, with all components on DM basis.

gum supplementation on apparent protein digestibility was assessed. Fermentation kinetics were studied by measuring hydrogen concentrations in the expired air as this expired gas originates solely from large-intestinal microbial fermentation $^{(16)}$. In the present paper, the methods of training the cats for sampling, and the procedure for measurement of the exhaled breath hydrogen are also described.

\section{Materials and methods}

\section{Animals}

Ten healthy adult domestic short-hair cats, with a mean body weight of 4.5 (SEM 0.5$) \mathrm{kg}$ and a mean age of 5.8 (SEM 2.7) years, were included in the present study. Five female and five male cats were used and all cats were castrated, except for one intact male. Before inclusion in the study, the cats underwent a thorough physical examination, and after an overnight fast, blood samples were taken for complete blood count and serum biochemistry analyses.

\section{Experimental design and diet}

The experiment was set up in a crossover design, with two periods of 5 weeks and two fibre supplements ( $4 \%$ guar gum; Vidogum GI, Unipektin Ingredients AG, cold viscosity: 3600-4500 mPas, hot viscosity: $4500-5300 \mathrm{mPas}$ ) or $4 \%$ cellulose (Arbocel BWW 40, Rettenmaier und Söhne) on a DM basis). The ten selected cats were randomly divided into two groups (group 1 and group 2). During the first $17 \mathrm{~d}$ of each period, the cats were fed twice a day (at 08.00 and 20.00 hours), while during the last $18 \mathrm{~d}$, they were fed four isoenergetic meals per day (at 06.00, 12.00, 18.00 and 24.00 hours). For $2 \mathrm{~d}$ between both periods, the cats were fed the experimental diet without fibre supplementation. All animals were individually fed and group housed with five cats per group in between meals. A commercially available dry cat food (Trovet Hypoallergenic lamb and rice; Netlaa bv) was fed at maintenance energy requirement ${ }^{(17)}\left(418.4 \mathrm{~kJ} / \mathrm{kg}^{0.67}\right.$ per $\mathrm{d}$, based on ideal body weight). This diet had a moderate protein content $(30 \cdot 1 \% \mathrm{DM})$ and rice was the only source of predominantly insoluble fibre. The analysed chemical composition and the ingredient composition specified by the manufacturer of the diet are shown in Table 1. Powdered supplements were first thoroughly mixed with $1 \mathrm{~g}$ of a commercially available canned diet (Hill's Prescription Diet Canine/ Feline a/d; Hill's Pet Nutrition, Inc.) to improve the supplement intake. Consecutively, this mixture was blended with the dry food by hand. Cats were weighed weekly and the amounts of feed were adjusted to maintain a stable body weight. Cats had ad libitum access to fresh drinking-water. The experimental protocol was approved by the Ethical Committee of the Faculty of Veterinary Medicine, Ghent University, Belgium (EC 2010/153; EC 2011/003) and was in accordance with institutional and national guidelines for the care and use of animals.

\section{Sampling}

Blood was collected via jugular venipuncture at $2 \mathrm{~h}$ after the first meal in week 5 of each period (four meals per $d$ ). Immediately after collection, blood samples were placed into vacutainer tubes containing lithium heparin. Additionally, in week 5 of each period, a total faecal collection was performed over a 5-d period, and on the last day fresh faecal samples were collected within 30 min of voiding. During faecal collection, cats were housed individually. Exhaled breath samples were collected as depicted in Fig. 1. The cats were trained once daily for this sample collection for a period of 3 months preceding the trial. An anaesthetic mask designed for cats was placed on the nose and mouth of the cat for increasing periods of time. At the end of the training period, the cats were sampled with minimal restraint and discomfort for $30-45 \mathrm{~s}$, as per the

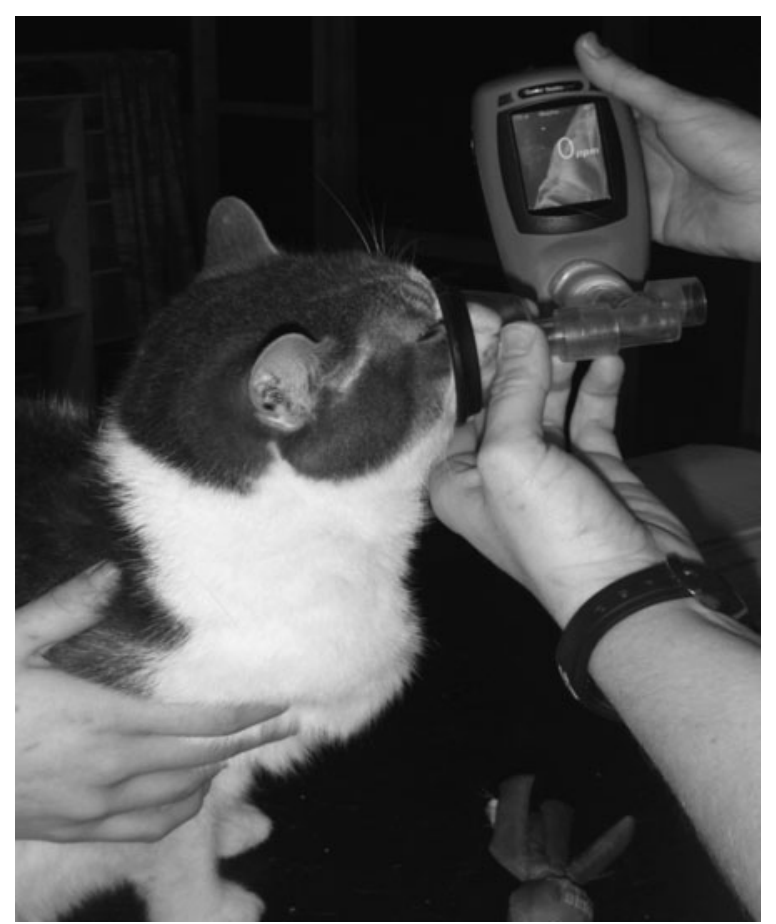

Fig. 1. Equipment and sampling technique for the hydrogen breath test used to study fermentation kinetics in nine cats fed a moderate-protein diet supplemented with guar gum (Vidogum GI, Unipektin Ingredients AG) or cellulose (Arbocel BWW 40, Rettenmaier und Söhne) in a 10-week crossover study. 
manufacturer's recommendation. The mask was connected to a hydrogen monitor ( $c f r$. infra). In week 3 of the first period (days 15-16), a pilot study was performed to establish the ideal measuring time points. All cats were sampled once prior to the morning meal and at every $30 \mathrm{~min}$ for $11.5 \mathrm{~h}$ postprandially to determine individual hydrogen concentrations over time. The resulting curves revealed that hourly sampling during $6 \mathrm{~h}$ was appropriate and this schedule was used in the main study during week 4 of each period. At each time point, two consecutive measurements were done 5 min apart and the means of both values were calculated. All measurements were repeated on two consecutive days.

\section{Chemical analyses}

The experimental diet was analysed for DM by drying to a constant weight at $103^{\circ} \mathrm{C}$ and for crude ash by combustion at $550^{\circ} \mathrm{C}$. Crude protein was calculated from Kjeldahl nitrogen $(6 \cdot 25 \times \mathrm{N}$, ISO $5983-1,2005)$, crude fat was analysed by the Soxhlet method (ISO 1443, 1973) and crude fibre by acidalkali digestion (ISO 5498, 1981). Nitrogen-free extract was calculated by subtracting crude ash, crude protein, crude fat and crude fibre on a DM basis from 100. Total dietary fibre was analysed as described by Prosky et al. ${ }^{(18)}$. Faecal $\mathrm{pH}$ was measured using a portable $\mathrm{pH}$ meter (Hanna Instruments Belgium). Additionally, faecal consistency was scored as described by Hesta et $a l .^{(19)}$. The SCFA were analysed using GC after extraction with diethyl ether ${ }^{(20)}$. The ammonia $(\mathrm{NH})_{3}$ was analysed by steam distillation and titration, as described by Bremner \& Keeney ${ }^{(21)}$. Indole, phenol and $p$-cresol were extracted using hexane and analysed using GC-MS/MS, as previously described ${ }^{(7)}$. Faecal samples of total collections were lyophilised and pooled per cat per period. Pooled faeces were sieved through a $1 \mathrm{~mm}$ mesh for hair removal, ground up in a grinding mill ( $1 \mathrm{~mm}$ mesh, Brabender Rotary Mill; Brabender GmbH \& Company KG) and proximate analyses were performed as described above. Bacterial nitrogen was analysed by the method of Mason ${ }^{(22)}$, with adaptations previously described by Hesta et al. ${ }^{(23)}$. The hydrogen concentration in the exhaled air samples was measured using a hydrogen monitor (Gastrolyzer; Bedfont Scientific). The electrochemical sensor sensitivity was 1 part per million (ppm) and the measurable concentration range was between 0 and 500 ppm. Before sampling, the hydrogen monitor was calibrated according to the manufacturer's instructions. Different calibration conditions (location, temperature, zeroing) were tested during the training period and pilot study, and the best calibration protocol was used during the test periods (zeroing and calibration in experimental room at $19^{\circ} \mathrm{C}$ ). Plasma acylcarnitine (free carnitine, acetyl-, propionyl-, butyryl- + isobutyryl-, isovaleryl- +2-methylbutyryl-, 3-hydroxy(OH)-isovaleryl-, 3-OH-butyryl-, tiglyl- + 3-methylcrotonyl-, methylmalonyl- and 3-OH-3-methylglutarylcarnitine (HMG)) and amino acid profiles (valine, leucine, methionine, phenylalanine, tyrosine, glycine, alanine, ornithine and citrulline) were analysed using LC-MS/MS, as previously described $^{(24)}$. Furthermore, plasma 1- and 3-methylhistidine were analysed, according to Spackman et al. ${ }^{(25)}$.

\section{Calculations}

The apparent protein digestibility coefficients were calculated using the following formula ${ }^{(26)}$ :

$$
\begin{gathered}
\text { Apparent protein digestibility }=((\text { nutrient intake } \\
- \text { nutrient excretion }) / \text { nutrient intake }) \times 100 \%
\end{gathered}
$$

\section{Statistical analysis}

For all data, normality of distribution was examined using the Kolmogorov-Smirnov test prior to further analyses. In the pilot study, the mean hydrogen concentration of all cats on the same supplement was calculated at each time point and these normally distributed data were further analysed using repeated measures ANOVA (time as within-subject factor, treatment as between-subject factor). Furthermore, area under the curve (AUC) was calculated for each cat and treatment effects were evaluated using the Student's independent samples $t$ test. All data from the main study were analysed in a univariate general linear model ANOVA for crossover designs to test the effects of period, treatment, group and cat nested in group. Day-to-day variability of the hydrogen measurements was tested using data from the main study, analysed in the same cats on consecutive days. The AUC was calculated for each cat for both days. Student's independent-samples $t$ tests were performed on the calculated AUC in both periods. For all analyses, Superior Performing Software Systems version 19 (SPSS, Inc.) was used. Statistical significance was set at $P<0.05$.

\section{Results}

\section{Energy intake and body weight}

The mean energy intake was compared for the last 2 weeks of each period, as sampling occured during these weeks. No treatment effect was found $(P=0 \cdot 25)$. One cat had to be excluded from the study due to continuous food refusal and weight loss. All cats that were supplemented with guar gum lost weight, while only one group lost weight when the cellulose-supplemented diet was fed. As a result, treatment $(P<0.01)$ and group $(P<0.01)$ effects were observed in the mean weight difference between the start and the end of each period (guar gum $-0.37 \mathrm{~kg}$; cellulose: $-0.01 \mathrm{~kg}$ ).

\section{Faecal parameters}

Faecal parameters are shown in Table 2. The faecal pH was lower in guar gum-supplemented cats compared to the cellulose-fed cats $(P<0 \cdot 01)$. No treatment effect was seen on the faecal consistency score, despite the numerically lower score (softer faeces) for guar gum-supplemented cats. Total faecal production over the 5 -d collection period and DM of the pooled faecal samples did not differ between both supplements $(P=0 \cdot 15$ and $P=0 \cdot 60$, respectively). Cellulosesupplemented cats tended to show higher faecal concentrations of acetic acid $(P=0.07)$, and with calculation of the ratio of faecal acetic acid:total SCFA, this treatment effect 
Table 2. Faecal characteristics and fermentation end products from nine cats fed a moderate protein diet supplemented with guar gum* or cellulose $\dagger$ in a 10 -week crossover study

\begin{tabular}{|c|c|c|c|c|c|c|c|c|}
\hline \multirow[b]{2}{*}{ Parameter } & \multicolumn{4}{|c|}{ Supplement } & \multicolumn{4}{|c|}{$P$} \\
\hline & GG & SEM & Cell & SEM & Treat. & Period & Group & Cat $\times$ group \\
\hline Faecal pH & $5 \cdot 26$ & 0.08 & $6 \cdot 55$ & 0.32 & $<0.01$ & 0.75 & 0.53 & 0.39 \\
\hline Faecal consistency scoreł & $2 \cdot 12$ & 0.39 & $2 \cdot 36$ & 0.38 & 0.16 & 0.42 & 0.01 & 0.07 \\
\hline Total faecal production $(\mathrm{g} / 5 \mathrm{~d})$ & $378 \cdot 33$ & 76.46 & 305.22 & 41.87 & 0.15 & $0 \cdot 16$ & 0.01 & 0.06 \\
\hline Faecal DM (\%) & $30 \cdot 82$ & 2.67 & 31.68 & 2.55 & 0.60 & 0.36 & 0.02 & 0.93 \\
\hline Acetic acid (mg/l) & 547.57 & $55 \cdot 78$ & 794.56 & $112 \cdot 70$ & 0.07 & 0.85 & 0.54 & 0.27 \\
\hline Propionic acid (mg/l) & $395 \cdot 35$ & 58.87 & $406 \cdot 31$ & 61.55 & 0.90 & 0.96 & $0 \cdot 18$ & 0.35 \\
\hline Butyric acid (mg/l) & 633.61 & 131.46 & $609 \cdot 81$ & $156 \cdot 23$ & 0.69 & 0.71 & 0.50 & $<0.01$ \\
\hline Isobutyric acid (mg/l) & $32 \cdot 70$ & 7.69 & $20 \cdot 15$ & 7.64 & $0 \cdot 10$ & 0.51 & 0.02 & 0.16 \\
\hline Isovaleric acid (mg/l) & 89.83 & $17 \cdot 11$ & 58.09 & $13 \cdot 84$ & 0.04 & 0.37 & 0.07 & 0.06 \\
\hline Valeric acid $(\mathrm{mg} / \mathrm{l})$ & 399.26 & 84.26 & 241.61 & 54.67 & 0.03 & 0.30 & $0 \cdot 13$ & 0.05 \\
\hline Total SCFA $(\mathrm{mg} / \mathrm{l}) \S$ & 2098.33 & 248.95 & 2130.54 & 331.79 & 0.95 & 0.61 & 0.20 & 0.04 \\
\hline Faecal $\mathrm{NH}_{3}(\mathrm{mg} / \mathrm{l})$ & $390 \cdot 83$ & $45 \cdot 41$ & $226 \cdot 65$ & 37.86 & 0.01 & 0.15 & 0.81 & 0.20 \\
\hline Indole (mg/l) & 0.36 & 0.13 & 0.06 & 0.02 & 0.02 & 0.22 & 0.14 & 0.39 \\
\hline$p$-Cresol $(\mathrm{mg} / \mathrm{l})$ & 1.57 & 0.29 & 0.50 & $0 \cdot 12$ & 0.01 & 0.08 & 0.12 & 0.26 \\
\hline Phenol (mg/l) & ND & & ND & & & & & \\
\hline
\end{tabular}

GG, guar gum; Cell, cellulose; Treat., treatment; ND, parameter could not be determined (below detection limit).

*Vidogum GI, Unipektin Ingredients AG.

†Arbocel BWW 40, Rettenmaier und Söhne.

$\ddagger$ Faecal consistency was scored on a five-point scale, as described by Hesta et al. ${ }^{(19)}$

$\S$ Total SCFA $=$ acetic + propionic + butyric + isobutyric + isovaleric + valeric acids.

reached statistical significance $(P=0 \cdot 01$, data not shown). Faecal propionic acid $(P=0.90)$, butyric acid $(P=0.69)$ and total SCFA $(P=0.95)$ concentrations did not differ between both fibre supplements. The faecal butyric acid:total SCFA ratio, however, tended to be higher for guar gumsupplemented cats $(P=0 \cdot 05)$. Faecal isovaleric acid $(P=0.04)$, valeric acid $(P=0.03)$, indole $(P=0.03), p$-cresol $(P=0.01)$ and $\mathrm{NH}_{3}(P=0 \cdot 01)$ concentrations were higher in guar gum-supplemented cats. In addition, the percentage of faecal bacterial nitrogen tended to be higher $(P=0.08)$ in cats fed the guar gum-supplemented diet (data not shown).

\section{Apparent protein digestibility coefficients}

The mean apparent protein digestibility coefficients in guar gum- and cellulose-supplemented cats were 71.8 (SEM 3.6) and 79.7 (SEM 1.0)\%, respectively, and tended to be lower in the guar gum-supplemented cats $(P=0 \cdot 07)$.

\section{Hydrogen concentrations}

Pilot study. The hydrogen concentration over time curves revealed a clear maximal hydrogen concentration in only one cat. The variability between cats was very high, as seen in Fig. 2. Numerically, the guar gum-supplemented cats showed a higher mean hydrogen concentration at all time points. However, no significant time $(P=0.20)$ or treatment $(P=0.20)$ effect could be observed. In one cat, the sampling could only be performed hourly and this cat was therefore excluded from the statistical analysis of the pilot study data (guar gum $n 5$; cellulose $n 4$ ). No treatment effect on the AUC of the hydrogen concentration over time curves was seen $(P=0 \cdot 20)$.

Main study. The mean hydrogen concentration per supplementation group for the six measured time points is shown in Fig. 3. The mean hydrogen concentration of guar gum-supplemented cats over all measured time points $(3 \cdot 20$ (sEm 1.05) ppm) was numerically higher than for cats supplemented with cellulose $(2 \cdot 11($ sem $0 \cdot 47) \mathrm{ppm})$. This difference, however, was not statistically significant $(P=0.325)$ and the variation between cats was very high. In both periods, no significant differences were found between AUC of all cats for two consecutive days $(P=0 \cdot 18$ for period 1 and $P=0 \cdot 72$ for period 2; data not shown).

\section{Plasma metabolites}

Plasma metabolites are shown in Table 3. No treatment effects were found for 1- and 3-methylhistidine, free carnitine, acetyl-, propionyl-, butyryl- + isobutyryl-, methylmalonyl-, 3-OH-3methylglutaryl-, tiglyl- + 3-methylcrotonyl- and 3-OH-isovalerylcarnitine. However, guar gum-supplemented cats showed higher 3-OH-butyrylcarnitine concentrations in plasma compared to cellulose-supplemented cats $(P=0 \cdot 02)$. For isovaleryl- + 2-methylbutyrylcarnitine, a trend towards higher plasma concentrations was observed when cats were supplemented with guar gum $(P=0 \cdot 09)$. Plasma leucine concentrations were lower $(P=0.02)$, and plasma valine $(P=0 \cdot 08)$ and phenylalanine $(P=0 \cdot 06)$ concentrations tended to be lower in guar gum-supplemented cats. On the contrary, guar gum-supplemented cats showed higher plasma alanine concentrations $(P=0 \cdot 02)$. For ornithine, citrulline, methionine, tyrosine and glycine, as well as the ratios methylmalonylcarnitine:valine, propionylcarnitine:methionine and HMG:leucine, no treatment effects were observed (data not shown).

\section{Discussion}

The present study was set up to investigate large-intestinal guar gum fermentation in domestic cats, with the emphasis on the amino acid-sparing potential of propionic acid. This mechanism was proposed by Verbrugghe et al. ${ }^{(6-8)}$ 


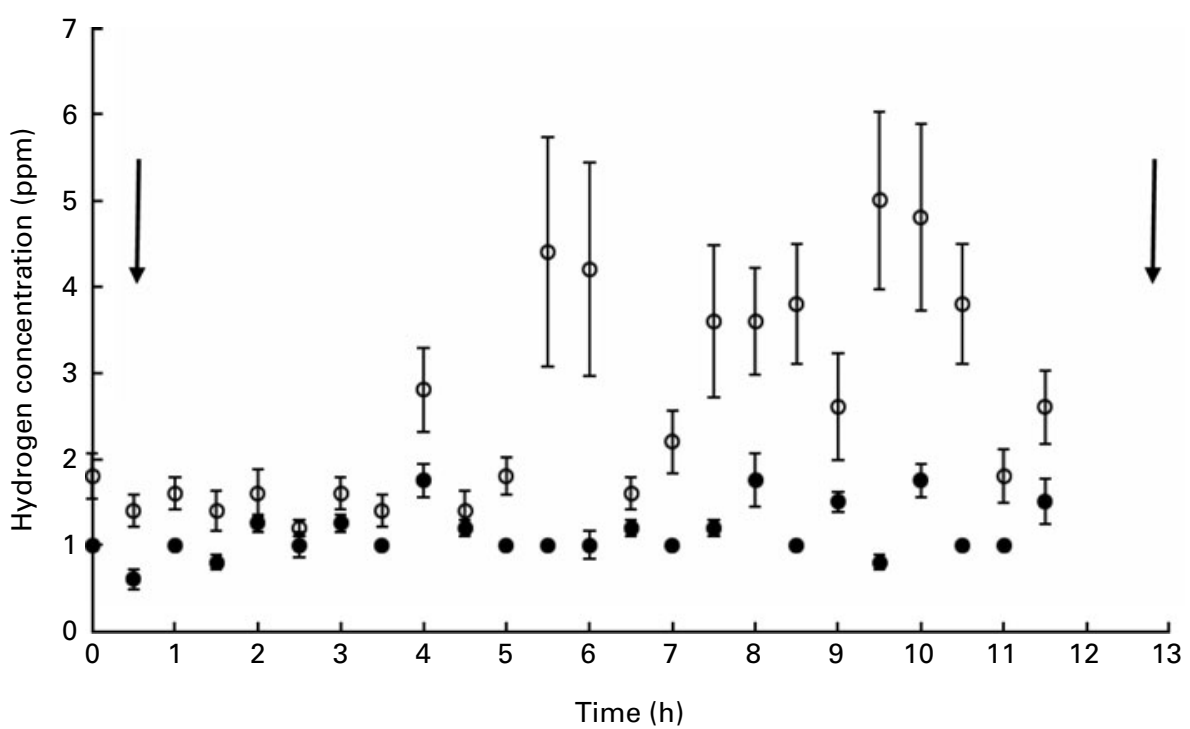

Fig. 2. Mean hydrogen concentration with their standard errors over time for nine cats fed a moderate-protein diet supplemented with guar gum (Vidogum Gl, Unipektin Ingredients AG; $O, n$ ) or cellulose (Vidogum GI, Unipektin Ingredients AG; $\bullet, n 4$ ) in the pilot experiment. The arrows indicate times of meal consumption. ppm, Parts per million.

upon supplementation of fructans in a commercially available canned cat food. To test this hypothesis, guar gum was supplemented to the experimental diet, as in vitro fermentation of this fibre source demonstrated high propionic acid concentrations $^{(4,5)}$. After $24 \mathrm{~h}$ of in vitro incubation with faecal inocula from donor cats fed different diets, guar gum yielded approximately $1 \mathrm{mmol}$ extra propionate per $\mathrm{g}$ of incubated organic matter compared to fructan fermentations ${ }^{(5)}$. In vitro data should be extrapolated very carefully to in vivo situations. Guar gum is completely indigestible by small-intestinal enzymes $^{(3)}$. In healthy adult cats, small-intestinal transit time is on average 2 to $3 \mathrm{~h}^{(27)}$, whereas total colon transit time is between 22 and $25 \mathrm{~h}^{(28)}$, depending on the measurement technique and diet of the cats. Therefore, a theoretical extra propionic acid production of $1 \mathrm{mmol} / \mathrm{g}$ of organic matter of guar gum might also be expected in vivo, within the large intestine. As concentrations of propionic acid in portal blood are assumed to be $1 / 1000$ th of the concentrations present in the colon and $80 \%$ of the portal blood concentration of propionic acid is metabolised in the liver ${ }^{(29)}$, every gram of organic matter of guar gum reaching the large intestine might provide $8 \times 10^{-4} \mathrm{mmol}$ of propionic acid to the gluconeogenic process. The present experiment aimed to confirm the amino acid-sparing hypothesis; however, further research for exact quantification of the amino acid-sparing potential of guar gum and propionic acid is still warranted.

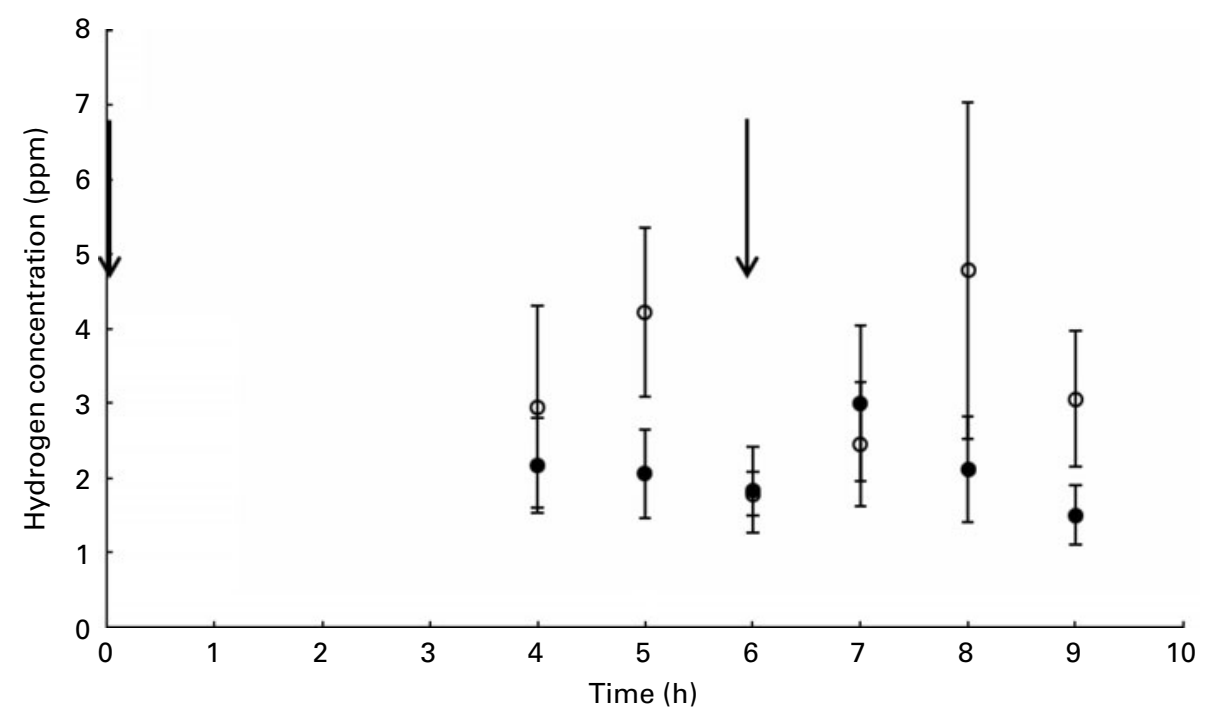

Fig. 3. Mean hydrogen concentrations with their standard errors at six time points measured in cats fed a moderate-protein diet supplemented with guar gum (Vidogum GI, Unipektin Ingredients AG; O, $n$ 9) or cellulose (Arbocel BWW 40, Rettenmaier und Söhne; $\bullet, n$ 9) in a 10-week crossover study. The arrows indicate two out of four meal consumption times. ppm, Parts per million. 
Table 3. Fermentation end product metabolites in plasma from nine cats fed a moderate protein diet supplemented with guar gum* or celluloset in a 10-week crossover study

\begin{tabular}{|c|c|c|c|c|c|c|c|c|}
\hline \multirow[b]{2}{*}{ Parameter } & \multicolumn{4}{|c|}{ Supplement } & \multicolumn{4}{|c|}{$P$} \\
\hline & GG & SEM & Cell & SEM & Treat. & Period & Group & Cat $\times$ group \\
\hline \multicolumn{9}{|l|}{ Plasma amino acid profile $(\mu \mathrm{mol} / \mathrm{l})$} \\
\hline Val & 24.96 & 1.80 & $27 \cdot 83$ & 3.02 & 0.08 & 0.39 & 0.28 & 0.05 \\
\hline Leu & 328.45 & 21.87 & 389.29 & $27 \cdot 34$ & 0.02 & 0.29 & 0.15 & $0 \cdot 12$ \\
\hline Met & 54.74 & $5 \cdot 76$ & $64 \cdot 20$ & $5 \cdot 84$ & 0.11 & 0.41 & 0.48 & 0.03 \\
\hline Phe & 61.55 & 3.54 & $71 \cdot 14$ & 2.51 & 0.06 & 0.41 & 0.62 & 0.28 \\
\hline Tyr & $44 \cdot 29$ & 3.55 & $45 \cdot 27$ & 2.45 & 0.82 & 0.57 & 0.05 & 0.23 \\
\hline Orn & 49.40 & 7.53 & $46 \cdot 33$ & 3.45 & 0.67 & 0.98 & 0.67 & 0.14 \\
\hline Cit & 34.44 & $4 \cdot 61$ & $40 \cdot 91$ & 3.41 & 0.23 & 0.89 & 0.12 & 0.31 \\
\hline Gly & $421 \cdot 34$ & $35 \cdot 24$ & 433.85 & $26 \cdot 56$ & 0.71 & 0.97 & 0.72 & 0.07 \\
\hline Ala & $840 \cdot 67$ & 74.04 & $642 \cdot 38$ & 64.55 & 0.02 & 0.73 & 0.63 & 0.05 \\
\hline \multicolumn{9}{|l|}{ Plasma acylcarnitine profile $(\mu \mathrm{mol} / \mathrm{l})$} \\
\hline Free carnitine & 23.07 & 1.58 & $18 \cdot 84$ & 2.06 & 0.14 & 0.04 & 0.20 & 0.48 \\
\hline Acetylcarnitine & 4.02 & 0.23 & 4.07 & 0.19 & 0.78 & 0.20 & 0.98 & 0.97 \\
\hline Propionylcarnitine & 0.44 & 0.08 & 0.26 & 0.04 & $0 \cdot 19$ & 0.53 & 0.51 & 0.98 \\
\hline Butyryl+isobutyrylcarnitine & 0.37 & 0.04 & 0.37 & 0.03 & $0 \cdot 11$ & 0.97 & 0.80 & 0.21 \\
\hline Methylmalonylcarnitine & 0.09 & 0.01 & 0.08 & 0.01 & 0.63 & 0.19 & 0.21 & 0.68 \\
\hline 3-OH-3-methylglutarylcarnitine & 0.01 & 0.00 & 0.02 & 0.00 & 0.21 & 0.59 & 0.78 & 0.70 \\
\hline Isovaleryl + 2-methylbutyrylcarnitine & 0.32 & 0.06 & 0.21 & 0.05 & 0.09 & 0.18 & 0.80 & $0 \cdot 11$ \\
\hline 3-OH-isovalerylcarnitine & 0.15 & 0.02 & 0.12 & 0.02 & 0.11 & 0.53 & 0.17 & 0.26 \\
\hline 3-OH-butyrylcarnitine & 0.07 & 0.01 & 0.04 & 0.01 & 0.02 & 0.67 & 0.68 & 0.69 \\
\hline Tiglyl + 3-methyl-crotonylcarnitine & $0 \cdot 10$ & 0.02 & 0.10 & 0.01 & 0.59 & 0.08 & 0.25 & 0.31 \\
\hline 3-Methylhistidine & 11.44 & 1.29 & $10 \cdot 26$ & 1.21 & 0.20 & 0.17 & 0.58 & $<0.01$ \\
\hline 1-Methylhistidine & 13.06 & 1.55 & 12.43 & 1.73 & 0.27 & 0.84 & 0.44 & $<0.01$ \\
\hline
\end{tabular}

GG, guar gum; Cell, cellulose; Treat., treatment; +, components cannot be separated.

*Vidogum GI, Unipektin Ingredients AG.

† Arbocel BWW 40, Rettenmaier und Söhne.

Due to the low propionic acid concentrations in peripheral blood, analysing this end product is only possible in portal blood. Likewise, measuring butyric acid in peripheral blood is impossible due to its nearly complete absorption and metabolism in colonocytes ${ }^{(30)}$. To circumvent difficult and invasive sampling techniques for measuring these SCFA, their respective carnitine esters can be analysed in plasma from the peripheral blood. Plasma acylcarnitine concentrations are representative of the mitochondrial acyl-CoA pool, and as a consequence, these parameters reflect metabolites available for the citric acid cycle $^{(7,31)}$. Additionally, some of the analysed plasma acylcarnitines represent metabolites of the catabolic pathway of, especially branched-chain, amino acids ${ }^{(32)}$. Large-intestinal and hepatic carbohydrate and amino acid metabolism can thus be assessed by studying such metabolites.

A potential problem arising with the use of guar gum in vivo is its high viscosity ${ }^{(3)}$. In previous in vitro work $^{(5)}$ and in the study described here, highly viscous guar gum was used. As the apparent protein digestibility tended to be lower in guar gum-supplemented cats, the gelling properties of the guar gum supplement have impaired protein digestion and absorption in the small intestine. Although plasma amino acid concentrations are not a direct measure of amino acid absorption, a significantly lower concentration of leucine and a trend towards lower valine and phenylalanine concentrations in plasma of guar gum-supplemented cats suggest decreased protein digestion and amino acid absorption from the small intestine. A higher concentration of L-alanine in plasma of guar gum-supplemented cats might indicate a higher amino acid turnover, as alanine is an important transport metabolite for amino groups in animals ${ }^{(32)}$.

Thus, a larger load of undigested protein and unabsorbed amino acids could have reached the large intestine in guar gum-supplemented cats, as a result of which more protein fermentation has occurred. This hypothesis was supported by higher concentrations of isovaleric and valeric acids, $\mathrm{NH}_{3}$, indole and $p$-cresol in faecal samples of guar gumsupplemented cats. In addition, guar gum might have stimulated microbial protein synthesis and turnover, as a tendency towards a higher percentage of bacterial nitrogen was observed in cats fed the guar gum-supplemented diet. In addition, in the plasma of these cats, a trend towards higher concentrations of isovaleryl- + 2-methyl-butyrylcarnitine was noted, which in turn suggests a higher microbial leucine and isoleucine degradation in the large intestine ${ }^{(32)}$. Besides isovaleric, isobutyric and valeric acids, $\mathrm{NH}_{3}$ and phenolic compounds, bacterial degradation of amino acids generally produces acetic and butyric acids, in proportions depending on the bacterial species composing the microbiota ${ }^{(33)}$. The extensive increase in protein fermentation in the large intestine could explain the absence of treatment effects on plasma propionylcarnitine and faecal propionic acid concentrations. These findings again highlight the difficulties of extrapolating in vitro data to in vivo situations. As plasma propionylcarnitine concentrations showed no treatment effect, the absorbed propionic acid concentrations were assumed to be similar for guar gum- and cellulose-supplemented cats. Therefore, no 
differences in hepatic amino acid and carbohydrate metabolism between treatments were expected. The conclusion was supported by the absence of treatment differences for plasma 1- and 3-methylhistidine, as both parameters were analysed in the present study as markers of endogenous protein catabolism $^{(34,35)}$.

An unexpected finding in the present experiment was a significantly higher concentration of 3-OH-butyrylcarnitine in plasma of guar gum-supplemented cats. In colonocytes, butyric acid can be converted to the ketone body 3-OH-butyryl$\mathrm{CoA}^{(36)}$, which can be measured in plasma as the carnitine ester 3-OH-butyrylcarnitine. As the ratio of faecal butyric acid: total SCFA was higher in guar gum-supplemented cats, a higher butyric acid production upon microbial fermentation of guar gum is suspected, explaining the treatment difference on 3-OH-butyrylcarnitine. This metabolite is partly converted to acetyl-CoA through the process of $\beta$-oxidation ${ }^{(36)}$, and partially absorbed into the bloodstream ${ }^{(37)}$. Likewise, acetyl-CoA can be absorbed from colonocytes into the bloodstream ${ }^{(37)}$. Acetyl-CoA is involved in many other metabolic pathways and is challenging to trace within the body. A concomitant increase in HMG was expected, as its CoA is a metabolite in the $\beta$-oxidation pathway ${ }^{(36)}$. Nonetheless, no differences in plasma HMG concentrations due to treatment could be observed, which might be due to the absorption of 3-OH-butyryl-CoA into the bloodstream and the conversion to acetoacetyl-CoA in the liver. Acetoacetyl-CoA can be converted in the liver to acetoacetate through pathways that do not involve the production of $\mathrm{HMG}^{(37)}$.

Besides studying the end product profile, fermentation kinetics of the fibre supplements were addressed using measurements of hydrogen concentrations in the exhaled breath. Reproducibility of the measurements was considered to be sufficient as no significant differences were found between the AUC calculated from measurements on two consecutive days for all cats, as has been shown in dogs ${ }^{(38)}$.

The main aim of the pilot experiment was to establish the time point at which maximal fermentation occurred in each cat, which was defined as the maximal expired hydrogen concentration. An estimation of the orocaecal transit time of the feed on both supplements could then also be made. However, this maximum concentration could only be clearly determined for one cat in the measurement period of $11.5 \mathrm{~h}$. The experimental protocol was changed following the pilot study, and the cats were fed four isoenergetic meals per $\mathrm{d}$ in the actual study starting from day 18 of each period. As such, a more consistent delivery of the fibre supplements to the gut microbiota was intended, and a constant fermentation of the guar gum supplement during the day was expected. However, data of the actual experiment did not reveal a significant treatment effect. Different hypotheses can be proposed for the absence of the expected postprandial rise of exhaled hydrogen concentrations in guar gum-supplemented cats in the pilot study and the absence of treatment effects in the actual experiment. One possible explanation is the increase in protein fermentation, as these processes are reported not to produce hydrogen ${ }^{(39)}$. Secondly, the extreme hydrogen-binding activity of guar gum, because of a large amount of hydroxyl groups in its chemical structure, might also explain the absence of a treatment effect $^{(1)}$. Furthermore, guar gum slows down small-intestinal transit due to its viscous nature in pigs ${ }^{(40)}$, humans ${ }^{(41)}$ and dogs ${ }^{(42)}$. In these experiments, the delay in small-intestinal transit time did not consist of several hours, so it cannot explain the absence of a hydrogen concentration increase during $11.5 \mathrm{~h}$ postprandially.

\section{Conclusions}

Guar gum fermentation did not evoke an increase in faecal propionic acid and plasma propionylcarnitine concentrations, suggesting a similar large-intestinal propionic acid production and absorption in guar gum- and cellulose-supplemented cats. The physicochemical properties of the gum have impaired the protein digestion and amino acid absorption within the small intestine, hence amino acid utilisation in cats' metabolism. Therefore, viscosity counteracts the amino acid-sparing potential of dietary fibre sources. Further experiments with partially hydrolysed guar gum of lower viscosity may be useful to potentiate the desirable in vivo side effects of this fibre supplement.

\section{Acknowledgements}

The present study was a part of the postgraduate study of the first author and was funded by the Institute for Promotion of Innovation through Science and Technology in Flanders (IWT, grant number 091050). The guar gum supplement was provided by Unipektin and the experimental diet was provided by Netlaa bv. K. R. was responsible for the study design, study performance, characteristic analysis, data analysis and manuscript drafting. M. H. and G. P. J. J., promoter and co-promoter of K. R., respectively, contributed to the development of the study design, data analysis and manuscript drafting. L. V. and B. W. supervised the plasma analyses and also contributed to the manuscript drafting. H. V. d. V. and A. V. collaborated in the study performance and contributed to the manuscript drafting. The authors also gratefully acknowledge Herman De Rycke for food and faecal sample analyses, Dirk Stockx and Greet Van de Velde for contributing to plasma analyses, Jurgen van Gool for plasma analyses and An Cools, Lien Bruynsteen, Jia Xu, Daisy Liu, Sarah Depauw, Eva Dhondt and Sanne Ott for technical assistance, Laura Statius and Saartje van Beirs for animal care taking, and Galena Quist-Rybachuk for proof reading. It is declared by the corresponding author that no conflict of interest exists for the present paper.

\section{References}

1. Chudzikowski RJ (1971) Guar gum and its applications. J Soc Cosm Chem 22, 43-60.

2. Fujioka R, Tanaka Y \& Yoshimura T (2009) Synthesis and properties of superabsorbent hydrogels based on guar gum and succinic anhydride. J Appl Polym Sci 114, 612-616.

3. Stewart ML \& Slavin JL (2006) Molecular weight of guar gum affects short-chain fatty acid profile in model intestinal fermentation. Mol Nutr Food Res 50, 971-976. 
4. Sunvold GD, Fahey GC, Merchen NR, et al. (1995) Dietary fiber for cats - in-vitro fermentation of selected fiber sources by cat fecal inoculum and in-vivo utilization of diets containing selected fiber sources and their blends. J Anim Sci 73, 2329-2339.

5. Rochus K, Janssens G, Bosch G, et al. (2010) In vitro evaluation of fibre and protein fermentation substrates in cats. In Proceedings of the WALTHAM International Nutritional Sciences Symposium, pp. 48. Cambridge: Cambridge University Press.

6. Verbrugghe A, Hesta M, Gommeren K, et al. (2009) Oligofructose and inulin modulate glucose and amino acid metabolism through propionate production in normal-weight and obese cats. Br J Nutr 102, 318-325.

7. Verbrugghe A, Janssens GPJ, Meininger E, et al. (2010) Intestinal fermentation modulates postprandial acylcarnitine profile and nitrogen metabolism in a true carnivore: the domestic cat (Felis catus). Br J Nutr 104, 972-979.

8. Verbrugghe A, Hesta M, Daminet S, et al. (2011) Propionate absorbed from the colon acts as gluconeogenic substrate in a strict carnivore, the domestic cat (Felis catus). J Anim Physiol Anim Nutr (Epublication ahead of print version 7 September 2011).

9. Kettelhut IC, Foss MC \& Migliorini RH (1980) Glucosehomeostasis in a carnivorous animal (cat) and in rats fed a high-protein diet. Am J Physiol 239, R437-R444.

10. Macdonald ML, Rogers QR \& Morris JG (1984) Nutrition of the domestic cat, a mammalian carnivore. Ann Rev Nutr 4 , $521-562$.

11. Morris JG (2002) Idiosyncratic nutrient requirements of cats appear to be diet-induced evolutionary adaptations. Nutr Res Rev 15, 153-168.

12. Kley S, Hoenig M, Glushka J, et al. (2009) The impact of obesity, sex, and diet on hepatic glucose production in cats. $A m \mathrm{~J}$ Physiol Regul Integr Comp Physiol 296, R936-R943.

13. Bunch SE (2003) Hepatobiliary diseases in the cat. In Small Animal Internal Medicine, 3rd ed., pp. 506-524 [RW Nelson and C Guillermo Couto, editors]. St Louis, MO: Mosby, Inc.

14. Polzin DJ \& Osborne CA (2000) Dietary management of feline chronic renal failure: where are we now? In what direction are we headed? J Feline Med Surg 2, 75-82.

15. Zoran DL (2002) The carnivore connection to nutrition in cats. J Am Vet Med Assoc 221, 1559-1567.

16. Papasouliotis K, Gruffydd-Jones TJ, Sparkes AH, et al. (1995) A comparison of orocecal transit times assessed by the breath hydrogen test and the sulphasalazine/sulphapyridine method in healthy Beagle dogs. Res Vet Sci 58, 263-267.

17. National Research Council (2006) Nutrient requirements and dietary nutrient concentrations. In Nutrient Requirements for Dogs and Cats, 2nd ed., pp. 366-367 [National Research Council, editor]. Washington, DC: The National Academies Press.

18. Prosky L, Asp NG, Furda I, et al. (1985) Determination of total dietary fibre in foods and food-products - collaborative study. J Assoc Off Anal Chem 68, 677-679.

19. Hesta M, Janssens GPJ, Debraekeleer J, et al. (2001) The effect of oligofructose and inulin on faecal characteristics and nutrient digestibility in healthy cats. J Anim Physiol Anim Nutr 85, 135-141.

20. Greenberg AE, Clesceri LS \& Eaton AD (1992) Aggregate organic constituents: organic and volatile acids. In Standard Methods for the Examination of Water and Wastewater, 18th ed., pp. 53-54 [AE Greenberg, LS Clesceri and AD Eaton, editors]. Washington, DC: American Public Health Association Publications.
21. Bremner JM \& Keeney RD (1965) Steam distillation methods for determination of ammonium, nitrate and nitrite. Anal Chem Acta 32, 485-495.

22. Mason V (1969) Some observations on the distribution and origin of nitrogen in sheep faeces. J Agric Sci 73, 99-111.

23. Hesta M, Roosen W, Janssens GPJ, et al. (2003) Prebiotics affect nutrient digestibility but not faecal ammonia in dogs fed increased dietary protein levels. Br J Nutr 90, 1007-1014.

24. Zytkovicz TH, Fitzgerald EF, Marsden D, et al. (2001) Tandem mass spectrometric analysis for amino, organic, and fatty acid disorders in newborn dried blood spots: a two-year summary from the New England newborn screening program. Clin Chem 47, 1945-1955.

25. Spackman DH, Stein WH \& Moore S (1958) Automatic recording apparatus for use in the chromatography of amino acids. Anal Chem 30, 1190-1206.

26. Cullison AE (1979) Apparent digestibility. In Feed and Feeding, 2nd ed., pp. 41-45 [AE Cullison, editor]. Reston, VA: Reston Publishing Company.

27. Chandler ML, Guilford WG \& Lawoko CRO (1997) Radiopaque markers to evaluate gastric emptying and small intestinal transit time in healthy cats. $J$ Vet Intern Med 11, 361-364.

28. Chandler ML, Guilford WG, Lawoko CRO, et al. (1999) Gastric emptying and intestinal transit times of radiopaque markers in cats fed a high-fiber diet with and without low-dose intravenous diazepam. Vet Radiol Ultrasound 40, $3-8$.

29. Cummings JH, Pomare EW, Branch WJ, et al. (1987) Short chain fatty acids in the human large intestine, portal, hepatic and venous blood. Gut 28, 1221-1227.

30. von Engelhardt W (1995) Absorption of short-chain fatty acids from the large intestine. In Physiological and Clinical Aspects of Short-chain Fatty Acids, 1st ed., pp. 149-169 [JH Cummings, JL Rombeau and T Sakata, editors]. Cambridge: Cambridge University Press.

31. Bremer J (1983) Carnitine - metabolism and functions. Physiol Rev 63, 1421-1466.

32. Michal G (1999) Amino acids and derivatives. In Biochemical Pathways: An Atlas of Biochemistry and Molecular Biology, 1st ed., pp. 47-48 [G Michal, editor]. New York: Wiley, Inc.

33. Macfarlane GT \& Gibson GR (1995) Microbiological aspects of the production of short-chain fatty acids in the large bowel. In Physiological and Clinical Aspects of Short-chain Fatty Acids, 1st ed., pp. 97-101 [JH Cummings, JL Rombeau and $\mathrm{T}$ Sakata, editors]. Cambridge: Cambridge University Press.

34. Sugawara T, Ito Y, Nishizawa N, et al. (2009) Measurement of the rate of myofibrillar protein degradation using the arteriovenous difference in plasma 3-methylhistidine concentration in rats. J Nutr Sci Vitaminol 55, 381-384.

35. Dragsted LO (2010) Biomarkers of meat intake and the application of nutrigenomics. Meat Sci 84, 301-307.

36. Roediger WEW (1995) The place of short-chain fatty acids in colonocyte metabolism in health and ulcerative colitis: the impaired colonocyte barrier. In Physiological and Clinical Aspects of Short-chain Fatty Acids, 1st ed., pp. 337-351 [JH Cummings, JL Rombeau and T Sakata, editors]. Cambridge: Cambridge University Press.

37. Henning S \& Hird FJR (1972) Ketogenesis from butyrate and acetate by cecum and colon of rabbits. Biochem $J \mathbf{1 3 0}$ $785-790$. 
38. Bissett SA, Spohr A, Guilford WG, et al. (1998) Reproducibility of breath hydrogen concentration measurements in dogs after change of diet. Am J Vet Res 59, 1523-1525.

39. Lu L, Xing D, Xie T, et al. (2010) Hydrogen production from proteins via electrohydrogenesis in microbial electrolysis cells. Biosens Bioelectron 25, 2690-2695.

40. Owusu-Asiedu A, Patience JF, Laarveld B, et al. (2006) Effects of guar gum and cellulose on digesta passage rate, ileal microbial populations, energy and protein digestibility, and performance of grower pigs. J Anim Sci 84, 843-852.

41. Jenkins DJA, Wolever TMS, Leeds AR, et al. (1978) Dietary fibres, fibre analogues, and glucose tolerance: importance of viscosity. Br Med J 1, 1392-1394.

42. Bueno L, Praddaude F, Fioramonti J, et al. (1981) Effect of dietary fibre on gastrointestinal motility and jejunal transittime in dogs. Gastroenterology 80, 701-707. 